Göteborg ITP preprint

hep-th/0007035

June, 2000

\title{
Manifestly Supersymmetric M-Theory
}

\section{Martin Cederwall, Ulf Gran, Mikkel Nielsen and Bengt E.W. Nilsson}

\author{
Institute for Theoretical Physics \\ Göteborg University and Chalmers University of Technology \\ SE-41296 Göteborg, Sweden
}

\begin{abstract}
In this paper, the low-energy effective dynamics of M-theory, eleven-dimensional supergravity, is taken off-shell in a manifestly supersymmetric formulation. We show that a previously proposed relaxation of the superspace torsion constraints does indeed accommodate a current supermultiplet which lifts the equations of motion corresponding to the ordinary second order derivative supergravity lagrangian. We comment on the relation and application of this completely general formalism to higher-derivative $\left(R^{4}\right)$ corrections. Some details of the calculation are saved for a later publication.
\end{abstract}

\footnotetext{
martin. cederwall@fy . chalmers. se

gran@fy.chalmers.se

mikkel@fy. chalmers.se

bengt.nilsson@fy.chalmers.se
} 
Cederwall, Gran, Nielsen, Nilsson: "Manifestly Supersymmetric M-Theory" $\ldots \ldots \ldots \ldots 2$

\section{INTRODUCTION}

One approach to probing M-theory at short distances is to consider the effective action beyond its lowest order approximation given by the second order (in \#(derivatives) + $\frac{1}{2} \#$ (fermions $)$ ) action $[1]$

$$
\begin{aligned}
-2 \kappa^{2} S= & \int d^{11} x \sqrt{-g}\left(R+\frac{1}{2 \cdot 4 !} H^{m n p q} H_{m n p q}\right)+\frac{1}{6} \int C \wedge H \wedge H \\
& + \text { terms with fermions }
\end{aligned}
$$

and investigate the higher-derivative corrections generated by the microscopic theory. Such corrections at order $R^{2}$ and $R^{4}$ have been extensively discussed in the literature, primarily in the context of string theory and ten-dimensional effective actions, but also in the elevendimensional context relevant to M-theory. The existence of these terms can be inferred by a variety of means in string theory, while in M-theory one must rely on anomaly cancellation arguments $[2,3]$, or (superparticle) loop calculations $[4,5,6,7,8]$ together with the connection to string theory and its effective action via dimensional reduction.

The methods used so far to deduce the existence of e.g. $R^{4}$ terms in eleven dimensions produce only isolated terms out of a large number of terms making up the complete superinvariant that it belongs to. It is of interest to have a better understanding of these superinvariants, and there has consequently been a lot of work invested into the supersymmetrisation of the isolated terms. In particular, $R^{2}$ and $R^{4}$ terms in ten dimensions were considered already some time ago, see ref. [9] and references therein. More recently also the $R^{4}$ term in eleven dimensions has been investigated [10] including a detailed study of superinvariants.

For these purposes it would be interesting to develop methods [11] based on superspace in eleven dimensions [12] that would incorporate supersymmetry in a manifest way. Although not yet developed into an easily applicable formalism, $N=1$ supergravity in ten dimensions has been constructed off-shell in terms of a linearised superspace lagrangian [13], including some superinvariants $[14,15]$, and should in principle lend itself to a complete analysis of superinvariants and deduction of the corresponding higher-derivative terms in ordinary component language. The situation in eleven-dimensional M-theory is, however, completely different due to the fact that an off-shell lagrangian formulation with a finite number of auxiliary fields is not known and may not even exist. From a general counting argument by Siegel and Roček [16] we know that this is true for $N=4$ super-Yang-Mills in four dimensions (and consequently also in ten dimensions) but that maximally supersymmetric supergravity passes the test. In higher dimensions, similar arguments were used in ref. [17] to prove that under some mild assumptions eleven-dimensional supergravity does in fact not allow for an 
off-shell lagrangian formulation. The analysis carried out here, when completed, will provide an independent check of that statement. In this respect the approach advocated here is parallel to the discussion of ten-dimensional super-Yang-Mills theory carried out in refs. [18] and [19], which does in fact prove that an off-shell lagrangian based on these superspace fields does not exist.

To implement the symmetries of any M-theory effective action in a manifest way, we will here follow ref. [11] and define the theory in superspace by means of the superspace Bianchi identities (SSBIs), which are integrability conditions when the theory is formulated in terms of superspace field strengths. From these we will derive consistency conditions on the form of the field equations. The analysis of the SSBIs will depend on the structure of certain components of the supertorsion, and one particular goal is to find connections between the various possible superinvariants and consistent expressions for the components of the supertorsion. The structure of these components, as e.g. which components can be set to zero under which conditions, will be clarified by our analysis. This is an important result since the torsion components are a vital input when proving $\kappa$-invariance for M2 and M5 branes coupled to background supergravity $[20,21,22]$ and M-theory corrected versions of it. In fact, one should compare to the situation in IIA and IIB string theory and the coupling to Dbranes $[23,24,25,26]$. Here it has been established that there are higher-derivative background field corrections also on the world-sheets of the branes, see e.g. refs. $[27,28]$ and references therein. The presence of such terms complicates the issue of $\kappa$-invariance and it becomes crucial to know the exact form of the supertorsion and to understand its relation to the corrections both in target space and on the brane.

Another aspect of the higher-derivative corrections is that it is to a large extent unclear how supersymmetry organises the infinite set of such terms into infinite subsets unrelated by supersymmetry. From previous work both in ten and eleven dimensions we know that adding one bosonic $R^{2}$ or $R^{4}$ term generates an infinite set of other terms of progressively higher order in number of derivatives. This is clear in any on-shell theory, as discussed in detail in the type IIB case in e.g. ref. [29]. In the heterotic case in ten dimensions an iterative procedure is needed even in the superspace treatment of the theory due to an implicit dependence on the three-form field strength in the supercurvature that appears in the SSBI, $d H=\operatorname{tr}\left(F^{2}-R^{2}\right)$, used to define the theory in superspace [3031]. This situation resembles the one for M-theory under discussion in this paper apart from the important fact that the corresponding SSBI for the four-form field strength is not added as a separate equation but will instead follow from the geometric SSBI for the supertorsion [32,33]. One might then suspect that if several linearised superinvariants exist at any given order, some appear as a result of iteration triggered by a lower order term, while some others, if they appear at all, generate new infinite series of terms. Of course, in order to determine which series of terms do actually occur, one has to invoke some microscopic description of the theory or rely on a 
comparison with string theory.

In this paper we will make use of the fact that any conceivable M-theory correction to the field equations must be compatible with supersymmetry and local Lorentz invariance. This is built into the SSBIs $[33,11]$ which when solved (the meaning of which is explained below) produce constraints on the supertorsion and other superfields that must be fulfilled by the corrections. As a first step we prove in this paper that the relaxed on-shell torsion constraints, argued for in ref. [11], are correct and do not lead to the field equations that follow from (1.1). This will done without specifying the auxiliary fields in terms of physical fields, making it possible to use the Weyl superspace introduced by Howe [33] to simplify the analysis of the standard on-shell theory. Once the auxiliary fields are related to physical fields, the role of Weyl superspace must be reconsidered, since the identification will involve a dimensionful parameter $\left(\alpha^{\prime 3}\right.$ for the $R^{4}$ term). This will be done elsewhere.

\section{RELAXED TORSION CONSTRAINTS}

One of the most important results proved in ref. [33] is that inserting the single constraint

$$
T_{\alpha \beta}^{c}=2 \Gamma_{\alpha \beta}^{c}
$$

used in the superspace construction of eleven-dimensional supergravity [12], into the SSBIs leads to the field equations corresponding to the lowest order lagrangian (1.1). This constraint must therefore be relaxed in such a way that the equations that then follow from the SSBIs are able to accommodate any higher-derivative correction terms to the field equations. In order to explain how this is done we need some details of the Weyl superspace formalism. This superspace is coordinatised by $z^{M}=\left(x^{m}, \theta^{\mu}\right)$ where $m$ enumerates the 11 bosonic and $\mu$ the 32 real fermionic coordinates respectively. The tangent superspace has as structure group the Lorentz group (not a superversion of it) times Weyl rescalings, and hence one introduces a supervielbein and a superconnection

$$
E_{M}^{A}(z), \quad \Omega_{M A}^{B}(z)=\omega_{M A}^{B}(z)+K_{M A}^{B}(z)
$$

where $\omega_{M A}{ }^{B}=\left(\omega_{M a}{ }^{b}, \frac{1}{4}\left(\Gamma^{a}{ }_{b}\right)_{\alpha}{ }^{\beta} \omega_{M a^{b}}{ }^{b}\right)$ is the Lorentz part and $K_{M A}{ }^{B}=\left(2 K_{M} \delta_{a}{ }^{b}, K_{M} \delta_{\alpha}{ }^{\beta}\right)$ the Weyl part, and the flat superindex $A=(a, \alpha)$ contains an $\mathrm{SO}(1,10)$ vector index $a$ and a (Majorana) spinor index $\alpha$. The super-two-form field strengths corresponding to the fields 
in (2.2) are (suppressing the wedge product symbol in the product of superforms)

$$
\begin{aligned}
& T^{A}=D E^{A}=d E^{A}+E^{B} \Omega_{B}{ }^{A}, \\
& R_{A}{ }^{B}=d \Omega_{A}{ }^{B}+\Omega_{A}{ }^{C} \Omega_{C}{ }^{B},
\end{aligned}
$$

and they satisfy the SSBI

$$
\begin{aligned}
& D T^{A}=E^{B} R_{B}{ }^{A}, \\
& D R_{A}{ }^{B}=0,
\end{aligned}
$$

of which only the first one will be used in this paper. Note that no separate superfield corresponding to the four-form field strength is introduced since both its Bianchi identity and field equation will emerge from the analysis of the torsion SSBI.

In order to obtain the form of the relaxed constraint given in ref. [11] we expand $T_{\alpha \beta}{ }^{c}$ in terms of irreducible tensors by means of the basis for symmetric $\Gamma$-matrices $\Gamma^{(1)}, \Gamma^{(2)}$, $\Gamma^{(5)}$, where $\Gamma^{(n)}$ indicate a product of $n$ antisymmetrised $\Gamma$-matrices with weight one, i.e.,

$$
T_{\alpha \beta}^{c}=2\left(\Gamma_{\alpha \beta}{ }^{d} X_{d}^{c}+\frac{1}{2} \Gamma_{\alpha \beta}{ }^{d_{1} d_{2}} X_{d_{1} d_{2}}{ }^{c}+\frac{1}{5 !} \Gamma_{\alpha \beta}{ }^{d_{1} \ldots d_{5}} X_{d_{1} \ldots d_{5}}{ }^{c}\right)
$$

with the understanding that the $X$ 's can be further decomposed into irreducible tensors. We will soon discuss each one of these irreducible $X$ tensors, but first we need to make a little digression into what is known as "conventional constraints" [34].

Conventional constraints are used to eliminate redundant superfields contained in the potentials of the theory, in the present case in the vielbeins and connections. They are imposed on tangent space tensors, here torsion components, and therefore have no effect on superspace reparametrisation invariance. We can distinguish between two types of constraints. The first type makes use of the arbitrariness in the distinction between spin connection and torsion (for each irreducible representation contained in the spin and Weyl connections) in eq. (2.3). This freedom must be fully utilised in order to obtain a solution of the spin connection in terms of the vielbeins. The second type of constraint makes use of arbitrary redefinitions of the vielbeins, $E^{A} \rightarrow E^{B} M_{B}{ }^{A}$, i.e., of the choice of tangent bundle, while the connection is unchanged. Once again, by using the torsion it is possible to impose conventional constraints in a gauge-covariant way. This second type of constraint reduces the field content of the supervielbein, and we must clearly limit its use to certain components in order not to lose the entire dynamics of the theory. An analysis of all the possible conventional constraints [11] will leave only $X$ 's in the representations 429 and $4290 \mathrm{in}(2.5)^{\dagger}$, as we will now discuss.

\footnotetext{
$\dagger$ The same statement appears without proof in ref. [35].
} 
Turning back to the components of the supertorsion at dimension 0 given in eq. (2.5), we note that $X_{d}^{c}$ decomposes into representations of dimension 65, with Dynkin label (20000), 55 with Dynkin label (01000), and 1 with Dynkin label (00000). Similarly, $X_{d_{1} d_{2}}{ }^{c}$ goes into 11 (10000), 165 (00100) and 429 (11000), and $X_{d_{1} \ldots d_{5}}{ }^{c}$ into 330 (00010), 462 (00002) and 4290 (10002). We use $M_{a}{ }^{b}$ to set $X_{a}{ }^{b}=\delta_{a}{ }^{b}$ and (part of) $M_{\alpha}{ }^{\beta}$ to set all antisymmetric tensors to zero. At this point we are left with the two fields which transform as 429 and 4290 under $\mathrm{SO}(1,10)$. The way these appear in the supertorsion, i.e., in $T_{\alpha \beta}{ }^{c}$, suggests a close connection to the M2 and M5 brane, respectively, for the 429 and 4290. Although it seems easier to deal with 429 we will in fact drop it and concentrate on the 4290 because of its probable relation to the anomaly canceling term related to the M5 brane. (The field of interest with dimension $0, \alpha^{\prime 3} W^{3}+\ldots$ ( $W$ is the Weyl tensor), does not contain the representation 429.) This will have to appear in the SSBI for the four-form superfield strength which hence will read $d * H=\frac{1}{2} H^{2}+X_{(8)}$, where $X_{(8)}$ is the eight-form polynomial in the curvature that was introduced in this context in ref. $[2,3]$. In this paper, however, we will not take the analysis this far but instead show that the relaxed torsion constraint [11]

$$
T_{\alpha \beta}^{c}=2\left(\Gamma_{\alpha \beta}^{c}+\frac{1}{5 !} \Gamma_{\alpha \beta}{ }^{d_{1} \ldots d_{5}} X_{d_{1} \ldots d_{5}}^{(4290) c}\right)
$$

is general enough to lift the field equations coming from (1.1).

More precisely, we will insert the following expanded irreducible torsion components

$$
\begin{aligned}
\operatorname{dim} \frac{1}{2}: T_{\alpha b}{ }^{c}= & S_{b}{ }^{c}{ }_{\alpha} \\
& +2\left(\Gamma_{(b} S_{d)}\right)_{\alpha} \eta^{c d} \\
T_{\alpha \beta} \gamma= & \frac{1}{120} \Gamma_{\alpha \beta}^{d_{1} \ldots d_{5}} Z_{d_{1} \ldots d_{5}}{ }^{\gamma} \\
& +\frac{1}{24} \Gamma_{\alpha \beta}^{d_{1} \ldots d_{5}}\left(\Gamma_{d_{1}} Z_{d_{2} \ldots d_{5}}\right)^{\gamma} \\
& +\frac{1}{12} \Gamma_{\alpha \beta}^{d_{1} \ldots d_{5}}\left(\Gamma_{d_{1} d_{2}} Z_{d_{3} d_{4} d_{5}}\right)^{\gamma} \\
& +\frac{1}{12} \Gamma_{\alpha \beta}^{d_{1} \ldots d_{5}}\left(\Gamma_{d_{1} d_{2} d_{3}} Z_{d_{4} d_{5}}\right)^{\gamma}+\frac{1}{2} \Gamma_{\alpha \beta}^{d_{1} d_{2}} Y_{d_{1} d_{2}}{ }^{c} \\
& +\frac{1}{24} \Gamma_{\alpha \beta}^{d_{1} \ldots d_{5}}\left(\Gamma_{d_{1} \ldots d_{4}} Z_{d_{5}}\right)^{\gamma}+\Gamma_{\alpha \beta}^{d_{\alpha} d_{2}}\left(\Gamma_{d_{1}} Y_{d_{2}}\right)^{\gamma} \\
& +\frac{1}{120} \Gamma_{\alpha \beta}^{d_{1} \ldots d_{5}}\left(\Gamma_{d_{1} \ldots d_{5}} Z\right)^{\gamma}+\frac{1}{2} \Gamma_{\alpha \beta}^{d_{1} d_{2}}\left(\Gamma_{d_{1} d_{2}} Y\right)^{\gamma}
\end{aligned}
$$

$\operatorname{dim} 1: \quad T_{a b}{ }^{c}=0$ 


$$
\begin{array}{rlr}
T_{a \beta} \gamma= & \frac{1}{24}\left(\Gamma^{d_{1} \ldots d_{4}}\right)_{\beta}{ }^{\gamma} A_{d_{1} \ldots d_{4} a}+\frac{1}{120}\left(\Gamma_{a} d_{1} \ldots d_{5}\right)_{\beta}{ }^{\gamma} A_{d_{1} \ldots d_{5}}^{\prime} & 2(00002) \\
& +\frac{1}{6}\left(\Gamma^{d_{1} d_{2} d_{3}}\right)_{\beta}{ }^{\gamma} A_{d_{1} d_{2} d_{3} a}+\frac{1}{24}\left(\Gamma_{a} d_{1} \ldots d_{4}\right)_{\beta}{ }^{\gamma} A_{d_{1} \ldots d_{4}}^{\prime} & 2(00010) \\
& +\frac{1}{2}\left(\Gamma^{d_{1} d_{2}}\right)_{\beta}{ }^{\gamma} A_{d_{1} d_{2} a}+\frac{1}{6}\left(\Gamma_{a} d_{1} d_{2} d_{3}\right)_{\beta}{ }^{\gamma} A_{d_{1} d_{2} d_{3}}^{\prime} & 2(00100) \\
& +\left(\Gamma^{d}\right)_{\beta}^{\gamma} A_{d a}+\frac{1}{2}\left(\Gamma_{a}{ }^{d_{1} d_{2}}\right)_{\beta}{ }^{\gamma} A_{d_{1} d_{2}}^{\prime} & 2(01000) \\
& +\left(\Gamma_{a}{ }^{d}\right)_{\beta}{ }^{\gamma} A_{d}^{\prime} & (10000) \\
& +\left(\Gamma_{a}\right)_{\beta}^{\gamma} A^{\prime} & (00000) \\
& +\frac{1}{120}\left(\Gamma^{d_{1} \ldots d_{5}}\right)_{\beta}{ }^{\gamma} B_{d_{1} \ldots d_{5}, a} & (10002) \\
& +\frac{1}{24}\left(\Gamma^{d_{1} \ldots d_{4}}\right)_{\beta}{ }^{\gamma} B_{d_{1} \ldots d_{4}, a} & (10010) \\
& +\frac{1}{6}\left(\Gamma^{d_{1} d_{2} d_{3}}\right)_{\beta}{ }^{\gamma} B_{d_{1} d_{2} d_{3}, a} & (10100) \\
& +\frac{1}{2}\left(\Gamma^{d_{1} d_{2}}\right)_{\beta}{ }^{\gamma} B_{d_{1} d_{2}, a} & \\
& +\left(\Gamma^{d}\right)_{\beta}^{\gamma} B_{d, a} &
\end{array}
$$

$$
\begin{aligned}
\operatorname{dim} \frac{3}{2}: T_{a b} \gamma= & t_{a b}{ }^{\gamma} \\
& +2\left(\Gamma_{[a} t_{b]}\right)^{\gamma} \\
& +\left(\Gamma_{a b} t\right)^{\gamma}
\end{aligned}
$$

(where, in addition to the conventional constraints used at dimension 0, we have used the ones corresponding to $\Omega_{M A}{ }^{B}$ and $M_{a}{ }^{*}{ }^{*}$ ) into the SSBIs and derive the spinor part of the spin $\frac{3}{2}$ field equation

$$
t_{\alpha} \sim\left(D^{3} X^{(4290)}\right)_{\alpha} .
$$

This will show that at the $\theta^{3}$ level in the auxiliary superfield $X^{(4290)}$, there is a spinor field that lifts the field equation $t_{\alpha}=0$ that would otherwise result from lagrangian in eq. (1.1). See e.g. ref. [37] for the relation between the Rarita-Schwinger field equation and $t_{\alpha}=0$ which in 11 dimensions reads $\Gamma^{a b c} T_{b c}=18 t^{a}-90 \Gamma^{a} t$.

\section{Solving the Superspace Bianchi identities OfF-Shell}

In this section we give a brief account of the steps required to derive the off-shell equation mentioned at the end of the last section. A more complete discussion will be presented elsewhere [38]. The method for solving the SSBI, $D T^{A}=E^{B} R_{B}{ }^{A}$, is to extract its component equations and solve these by increasing dimension. The equation of lowest dimension, $\frac{1}{2}$, is the one multiplying the three-form $E^{\alpha} E^{\beta} E^{\gamma}$ and with $A=a$,

$$
0=R_{(\alpha \beta \gamma)}{ }^{d}=D_{(\alpha} T_{\beta \gamma)}{ }^{d}+T_{(\alpha \beta}^{E} T_{|E| \gamma)}{ }^{d}
$$

\footnotetext{
* In ref. [36], it was proposed that a spinor superfield should be used to take the theory off-shell. This field was subsequently shown to be eliminated by a conventional constraint corresponding to $M_{a}{ }^{\beta}$ [33], and does not occur in our expansion of the torsion.
} 
where $(\ldots)$ indicates symmetrisation of the indices (except for the ones between bars $|\ldots|$ ). This equation can be decomposed into a large number of equations, each one corresponding to an irreducible tensor appearing in the decomposition of the symmetric product of three spinors times a vector, which is the tensor structure of the SSBI (3.1). When the expansions of the torsion components are inserted into these irreducible tensor equations, all irreducible tensor parts of the torsion will drop out except the ones that coincide with the representation specifying the equation.

We should also mention that we restrict ourselves to a linearised analysis. A more nonlinear treatment is feasible, at least in the original fields, but here the ordinary supergravity fields and the auxiliary ones are treated on equal footing. In this paper we also neglect vector derivatives on the auxiliary superfield $X^{4290}$.

Since the equation (3.1) involves the fields at $\theta$ level in $X^{(4290)}$, we need to expand also $D_{\alpha} X_{a_{1} \ldots a_{5}}{ }^{b}$ into irreducible tensors. This gives rise to the following spinorial tensors:

$\begin{array}{llll}(10001) & \tilde{X}_{a}^{\alpha} & (20001) & \tilde{X}_{a, b}^{\alpha} \\ (01001) & \tilde{X}_{a_{1} a_{2}}^{\alpha} & (11001) & \tilde{X}_{a_{1} a_{2}, b}^{\alpha} \\ (00101) & \tilde{X}_{a_{1} a_{2} a_{3}}^{\alpha} & & \\ (00011) & \tilde{X}_{a_{1} \ldots a_{4}}^{\alpha} & & \\ (00003) & \tilde{X}_{a_{1} \ldots a_{5}}^{\alpha} & (10003) & \tilde{X}_{a_{1} \ldots a_{5}, b}^{\alpha}\end{array}$

where the $\tilde{X}$ 's with $a_{1} \ldots a_{n}$ are $\Gamma$-traceless $n$-forms and the ones with $a_{1} \ldots a_{n}, b$ are $\Gamma$ traceless tensors with $n$ antisymmetric indices and $\tilde{X}_{\left[a_{1} \ldots a_{n}, b\right]}=0=\tilde{X}_{a_{1} \ldots a_{n}, b} \eta^{a_{n} b}$ (note the distinction between these two kinds of representation, kept track of by the comma between the $a_{i}$ indices and the $b$ index). The solution to the SSBI of dimension $\frac{1}{2}$ then relates the various irreducible tensors in the supertorsion to the $\tilde{X}$ 's in (3.2). In fact, as a consequence of using Weyl superspace, with the extra conventional constraints associated with the Weyl connection, we find that the torsions involved in this SSBI are uniquely determined by the $\tilde{X}$ 's above. Thus if we set $X^{(4290)}$ to zero these torsions will vanish without invoking any extra assumptions, a result that also follows from the work of Howe in ref. [33]. Besides these relations, the solution also tells us that $\tilde{X}_{a, b}^{\alpha}$ and $\tilde{X}_{a_{1} \ldots a_{5}, b}^{\alpha}$ are zero (the former one only when the representation 429 is left out of the dimension 0 torsion). In this paper, we spare the reader from the exact expression for the torsion components in terms of $X^{(4290)}$. The calculation involves a certain degree of technical complexity, which is left for ref. [38].

We now turn to the SSBIs with dimension 1 . There are two such equations, namely

$$
\begin{aligned}
& R_{\alpha \beta c}{ }^{d}=2 D_{(\alpha} T_{\beta) c}{ }^{d}+D_{c} T_{\alpha \beta}{ }^{d}+T_{\alpha \beta}{ }^{E} T_{E c}{ }^{d}+2 T_{c(\alpha}{ }^{E} T_{|E| \beta)}{ }^{d}, \\
& R_{(\alpha \beta \gamma)}{ }^{\delta}=D_{(\alpha} T_{\beta \gamma)}{ }^{\delta}+T_{(\alpha \beta}{ }^{E} T_{|E| \gamma)}{ }^{\delta} .
\end{aligned}
$$


To deal with these equations, we must expand the superfield $X^{(4290)}$ at the $\theta^{2}$ level, and take into account the results already obtained at $\theta$ level. As mentioned above, we aim at showing that the introduction of the auxiliary fields generates a right-hand side of the spinor part of the equation of motion for the gravitino field. At present, we therefore only need to consider the irreducible tensors at dimension 1 whose spinorial derivative contains a spinor. These are the forms, denoted $A$ and $A^{\prime}$ in eq. (2.7). Since now the curvatures entering eq. (3.3) are nonzero taking values in the structure group, they must be eliminated. From the $R_{\alpha \beta c}{ }^{d}$ we get the information that the symmetric traceless part in $c d$ has to vanish, and the rest are used to eliminate $R_{\alpha \beta \gamma}{ }^{\delta}$ by the structure group condition. In contrast to the equations at dimension $\frac{1}{2}$, where the full representation content of the index structure of the SSBI made impact on the fields (to the extent that the representations were present at level $\theta$ in $X^{(4290)}$ ), some equations now turn out to be linearly dependent. A naive counting of fields and equations fails, and, as we will see, this is absolutely essential in order for the auxiliary superfield to contain components entering the equations of motion. This exceptional behaviour relies on the exact form of the solutions at dimension $\frac{1}{2}$, and comes at work for the three-forms, where three equations reduce to two, and for the four-forms, where all three equations are identical. The zero-, one-, two- and five-forms at second level in $X$ are set to zero (modulo terms $\sim D_{b} X_{a_{1} \ldots a_{5}}{ }^{b}$ in the five-forms), and the relevant surviving part is parametrised as

$$
\begin{aligned}
& \frac{1}{10} D_{[\alpha} D_{\beta]} X_{a_{1} \ldots a_{5}, b} \\
& =\Gamma_{\left[a_{1} a_{2} a_{3}\right.}{ }^{e} V_{\left.a_{4} a_{5}\right] b e}+\Gamma_{b\left[a_{1} a_{2}\right.}{ }^{e} V_{\left.a_{3} a_{4} a_{5}\right] e}-\frac{6}{7} \eta_{b\left[a_{1}\right.} \Gamma_{a_{2} a_{3}}{ }^{e_{1} e_{2}} V_{\left.a_{4} a_{5}\right] e_{1} e_{2}} \\
& +\Gamma_{a_{1} \ldots a_{5}}{ }^{e_{1} e_{2} e_{3}} W_{b e_{1} e_{2} e_{3}}+\Gamma_{b\left[a_{1} \ldots a_{4}\right.}{ }^{e_{1} e_{2} e_{3}} W_{\left.a_{5}\right] e_{1} e_{2} e_{3}}-\frac{6}{7} \eta_{b\left[a_{1}\right.} \Gamma_{\left.a_{2} \ldots a_{5}\right]}{ }^{e_{1} \ldots e_{4}} W_{e_{1} \ldots e_{4}} \\
& +\Gamma_{\left[a_{1} a_{2} a_{3}\right.} V_{\left.a_{4} a_{5}\right] b}+\Gamma_{b\left[a_{1} a_{2}\right.} V_{\left.a_{3} a_{4} a_{5}\right]}-\frac{6}{7} \eta_{b\left[a_{1}\right.} \Gamma_{a_{2} a_{3}}{ }^{e} V_{\left.a_{4} a_{5}\right] e} \\
& +\Gamma_{a_{1} \ldots a_{5}}{ }^{e_{1} e_{2}} W_{b e_{1} e_{2}}+\Gamma_{b\left[a_{1} \ldots a_{4}\right.}{ }^{{ }^{e} e_{2}} W_{\left.a_{5}\right] e_{1} e_{2}}-\frac{6}{7} \eta_{b\left[a_{1}\right.} \Gamma_{\left.a_{2} \ldots a_{5}\right]}{ }^{e_{1} e_{2} e_{3}} W_{e_{1} e_{2} e_{3}} \\
& +\ldots
\end{aligned}
$$

with the relations

$$
A+2 A^{\prime}+\frac{2 \cdot 5}{7 \cdot 11 \cdot 23}\left(547 V+2^{5} \cdot 3^{3} \cdot 17 W\right)=0
$$

for the four-forms, and

$$
\begin{aligned}
& A=-\frac{2^{2}}{3 \cdot 11 \cdot 23}\left(89 \mathrm{~V}+2^{2} \cdot 3 \cdot 5 \cdot 139 \mathrm{~W}\right), \\
& A^{\prime}=\frac{2^{3}}{3 \cdot 11 \cdot 23}(2 \cdot 47 \mathrm{~V}-3 \cdot 5 \cdot 41 \mathrm{~W})
\end{aligned}
$$

for the three-forms. The linear dependence already mentioned makes us confident in these expressions. In the unmodified supergravity $(V=W=0)$, one four-form in the dimension 
1 torsion survives, and is identified with the four-form field strength $H$. In the present situation, it is a priori not obvious which combination of the three surviving four-forms that should be identified with this physical field (the criterion being that it is closed), and the answer to this question will have to await the solution of the SSBIs at dimension 2.

One further result at dimension 1 is that the Weyl part of the curvature, $G_{\alpha \beta}=$ $\frac{1}{32} R_{\alpha \beta \gamma}{ }^{\gamma}$, vanishes, as was shown in ref. [33] for the unmodified supergravity. This is a very positive sign, since it indicates that the theory even with the relaxed torsion constraints we have used to take it off-shell is equivalent to one with only the Lorentz group as structure group as discussed by Howe [33]. We will comment on this further in the concluding section.

Finally, we consider the SSBIs at dimension $\frac{3}{2}$, which read

$$
\begin{aligned}
& 2 R_{\alpha[b c]}^{d}=D_{\alpha} T_{b c}{ }^{d}+2 D_{[b} T_{c] \alpha}{ }^{d}+2 T_{\alpha[b}{ }^{E} T_{|E| c]}{ }^{d}+T_{b c}{ }^{E} T_{E \alpha}{ }^{d} \\
& 2 R_{a(\beta \gamma)}{ }^{\delta}=D_{a} T_{\beta \gamma}{ }^{\delta}+2 D_{(\beta} T_{\gamma) a}{ }^{\delta}+2 T_{a(\beta}{ }^{E} T_{|E| \gamma)}{ }^{\delta}+T_{\beta \gamma}{ }^{E} T_{E a}{ }^{\delta}
\end{aligned}
$$

In an unconstrained superfield in the representation 4290, there are two spinors at level $\theta^{3}$. The index structure of the SSBIs at this level also contains two spinor equations. We have to take into account what has been learned about $X^{(4290)}$ at lower levels. Specifically, at dimension 1 some of the antisymmetric tensors containing a spinor at the next level vanished. Miraculously, again, all of these go into the same linear combination, while the spinor coming from the three- and four-forms survives. One spinor thus remains, and goes into part of the field equation for the Rarita-Schwinger field, which then reads

$$
t_{\alpha}=\frac{17}{2^{2} \cdot 3^{3} \cdot 5^{2} \cdot 7 \cdot 11 \cdot 13 \cdot 61}\left(\Gamma^{b_{1} b_{2} b_{3}}\right)^{\beta \gamma}\left(\Gamma^{b_{4} b_{5} a}\right)_{\alpha}{ }^{\delta} D_{[\beta} D_{\gamma} D_{\delta]} X_{b_{1} \ldots b_{5}, a}
$$

Also the spinor component of the Weyl curvature, $G_{\alpha}=\frac{1}{32}\left(\Gamma^{a}\right)_{\alpha}{ }^{\beta} R_{a \beta \gamma}{ }^{\gamma}$, is set to zero.

These calculations involve some rather heavy $\Gamma$-matrix algebra which has been facilitated enormously by the development of a Mathematica based program [39]. In particular, the results in this paper rely on a large number of Fierz identities which, as explained below, can be completely systematised. By using the algebraic program to compute some small number of final coefficients, any computation requiring Fierzing is easily dealt with. The systematisation and use of the program is most clearly explained through an example: Consider the two spinors appearing at $\theta^{3}$ level in the superfield $X^{4290}$. We would like to know how these two spinors are related via supersymmetry to the three- and four-forms at level $\theta^{2}$. For this one needs to discuss the Fierz identities for structures of the kind $\left(\Gamma^{a b \ldots}\right)_{[\alpha \beta}\left(\Gamma^{c d \ldots}\right)_{\gamma] \delta}$ where the vector indices define the representation 4290 and $[\ldots]$ means antisymmetrisation. There are exactly seven such structures differing in the way the vector indices are distributed. However, since the representation 4290 occur exactly twice in the 
product of a spinor with three antisymmetrised spinors, there must be precisely five linearly independent Fierz identities between the seven $\Gamma \Gamma$ structures. Or in other words, by picking two of the structures as independent, the others are expressible as linear combinations of these. The corresponding coefficients are then computed by contracting these relations with a basis of $\Gamma$ matrices using the algebraic program to perform the resulting algebra. Using this technique it is easy to disentangle all the information hidden in the various SSBIs discussed in this paper.

\section{Conclusions}

We have demonstrated that, through a series of seemingly miraculous numerical coincidences (which, however, due to the similarities with ten dimensions [40], both regarding the constraints and the subsequent manipulations of the SSBIs, one would strongly expect to occur), the relaxation of the torsion constraint at dimension zero is capable of accommodating an offshell formulation. By off-shell we here simply mean that the equations of motion from (1.1) are relaxed by the introduction of a current supermultiplet, contained in the supertorsion along with the supergravity multiplet. In this sense, the term "on-any-shell" might be more appropriate. However, since the non-existence of an off-shell action has to our knowledge not been proven, the possibility is not ruled out that the auxiliary fields produced by this formalism are the correct ones for the construction of such an action. We would like to stress that since the degrees of freedom contained in the eleven-dimensional supergravity multiplet describe only low-energy effective dynamics of M-theory, and this system is not supposed to be subject to quantisation, the absence of an action at this level is completely acceptable. The results are sofar partial. We have not yet investigated all equations of motion. In a following paper [38], we will give a more detailed account of the calculations.

An obvious application of the formalism, as mentioned in the introduction, is to use it to derive higher-derivative corrections to M-theory, beginning with $R^{4}$ terms and their superpartners. The identification of our auxiliary field $X^{(4290)}$ as a supergravity self-interaction clearly breaks Weyl invariance. It is encouraging to note that the corresponding curvatures vanish, as far as our analysis goes, which indicates that the correct procedure is to restrict to the Lorentz structure group in order to avoid ambiguities in the definition of Weyl weights, while retaining the corresponding conventional constraints.

Brane dynamics in general backgrounds is most conveniently described in terms of quantities pulled back from target superspace to the world-volume. It is known that $\kappa$ symmetry quite generally demands the background fields to be on-shell. This must still be true for branes in backgrounds modified by higher-derivative corrections. We believe that our formalism will be essential for such an analysis. One question arises directly: Is the 
action for e.g. the M2-brane still given by the same expression,

$$
S \sim \int d^{3} \sigma \sqrt{-g}+\int C
$$

so that the corrections come only through the pullbacks of the modified background fields, or is this form changed? We have not discussed the superspace tensor fields in this paper, but by analysing the dimension zero identity, one realises that the equation $d H=0$ demands $H$ to have non-vanishing components even at negative dimensions. These will appear in a $\kappa$-transformation of the WZ term in eq. (4.1), but do not have any torsion counterpart to cancel. We hope to be able to come back also to this issue.

Finally, it would also be interesting to investigate in a strict sense whether the assumption of locality, which is implicit in our work, limits the current multiplet to self-interactions

of the supergravity multiplet or whether there are traces of interactions with other M-theory states.

Acknowledqements: The authors are grateful to Kasper Peeters, Pierre Vanhove and Anders Westerberg for very inspiring discussions and for their generous communication of work in progress [10]. This work is partly supported by EU contract HPRN-CT-2000-00122 and by the Swedish and Danish Natural Science Research Councils.

For some representation theoretical considerations, the program LiE [41] has been useful.

\section{REFERENCES}

[1] E. Cremmer, B. Julia and J. Sherk, "Supergravity theory in eleven-dimensions", Phys. Lett. B76 (1978) 409 .

[2] C. Vafa and E. Witten, "A one loop test of string duality", Nucl. Phys. B447 (1995) 261 hep-th/9505053.

[3] M.J. Duff, J.T. Liu and R. Minasian, "Eleven-dimensional origin of string-string duality: a one loop test", Nucl. Phys. B452 (1995) 261 hep-th/9506126.

[4] M.B. Green, M. Gutperle and P. Vanhove, "One-loop in eleven dimensions", Phys. Lett. B409 (1997) 177 hep-th/9706175.

[5] J.G. Russo and A.A. Tseytlin, "One-loop four-graviton amplitude in eleven-dimensional supergravity", Nucl. Phys. B508 (1997) 245 hep-th/9707134.

[6] M.B. Green. M. Gutperle and H.-h. Kwon, " ${ }^{16}$ and related terms in M-theory on $T^{2}$ ", Phys. Lett. B421 (1998) 149 hep-th/9710151.

[7] M.B. Green, M. Gutperle and H.-h. Kwon, "Light-cone quantum mechanics of the eleven-dimensional superparticle", J. High Energy Phys. o8 (1999) o12 hep-th/9907155.

[8] M.B. Green, H.-h. Kwon and P. Vanhove, "Two loops in eleven dimensions", Phys. Rev. D61 (2000) 104010 hep-th/9910055. 
Cederwall, Gran, Nielsen, Nilsson: "Manifestly Supersymmetric M-Theory" ........ 13

[9] M. de Roo, H. Suelmann and A. Wiedemann, "The supersymmetric effective action of the heterotic string", Nucl. Phys. B405 (1993) 326 [hep-th/9210099].

[10] K. Peeters, P. Vanhove and A. Westerberg, in preparation.

[11] B.E.W. Nilsson, "A supersymmetric approach to branes and supergravity", in "Theory of elementary particles", Proc. of the 31st international symposium Ahrenshoop, September 2-6, 1997, Buckow, Eds H. Dorn et al. (Wiley-VCH 1998), Göteborg-ITP-g8-o9 hep-th/0007017.

[12] L. Brink and P. Howe, "Eleven-dimensional supergravity on the mass-shell in superspace", Phys. Lett. B91 (1980) 384; E. Cremmer and S. Ferrara, "Formulation of eleven-dimensional supergravity in superspace", Phys. Lett. B91 (1980) 61.

[13] P. Howe, H. Nicolai and A. van Proeyen, "Auxiliary fields and a superspace lagrangian for linearized ten-dimensional supergravity", Phys. Lett. B112 (1982) 446.

[14] R. Kallosh, "Strings and superspace", in "Unification of fundamental interactions", Proc. of Nobel Symposium 67, Marstrand, Sweden, June 2-7, 1998, Eds L. Brink et al, Physica Scripta T15.

[15] B.E.W. Nilsson and A. Tollstén, "Supersymmetrization of $\zeta(3) R^{4}$ in superstring theory", Phys. Lett. B181 (1986) 63 .

[16] M. Roček and W. Siegel, "On off-shell multiplets", Phys. Lett. B105 (1981) 275.

[17] V.O. Rivelles and J.G. Taylor, "Off-shell no go theorems for higher dimensional supersymmetries and supergravities", Phys. Lett. B121 (1983) 37.

[18] B.E.W. Nilsson, "Off-shell fields for the 10-dimensional supersymmetric Yang-Mills theory", GöteborgITP-81-6.

[19] B.E.W. Nilsson, "Pure spinors as auxiliary fields in the ten-dimensional supersymmetric Yang-Mills theory", Class. Quant. Grav. 3 (1986) L41.

[20] E. Bergshoeff, E. Sezgin and P.K. Townsend, "Supermembranes and eleven-dimensional supergravity", Phys. Lett. B189 (1987) 75; "Properties of the eleven-dimensional supermembrane theory", Ann. Phys. 185 (1988) 330.

[21] M.J. Duff, P.S. Howe, T. Inami and K.S. Stelle, "Superstrings in D=10 from supermembranes in D=11", Phys. Lett. B191 (1987) 70.

[22] M. Cederwall, B.E.W. Nilsson and P. Sundell, "An action for the super-5-brane in D=11 supergravity", J. High Energy Phys. o4 (1998) oo7, hep-th/9712059].

[23] M. Cederwall, A. von Gussich, B.E.W. Nilsson and A. Westerberg, "The Dirichlet super-three-brane in ten-dimensional type IIB supergravity", Nucl. Phys. B49o (1997) 179 hep-th/961014q.

[24] M. Aganagić. C. Popescu, J.H. Schwarz, "D-brane actions with local kappa symmetry", Phys. Lett. B393 (1997) 311 hep-th/9610249.

[25] M. Cederwall, A. von Gussich, B.E.W. Nilsson, P. Sundell and A. Westerberg, "The Dirichlet super-pbranes in ten-dimensional type IIA and IIB supergravity", Nucl. Phys. B49o (1997) 163 hep-th/9611159.

[26] E. Bergshoeff and P.K. Townsend, "Super D-branes", Nucl. Phys. B49o (1997) 145 hep-th/9711173.

[27] C.P. Bachas, P. Bain and M.B. Green, "Curvature terms in the D-brane actions and their M-theory origin", J. High Energy Phys. o5 (1999) 11 hep-th/9903210.

[28] N. Wyllard, "Derivative corrections to D-brane actions with constant background fields", hep-th/0008125.

[29] M.B. Green and S. Sethi, "Supersymmetry constraints on type IIB supergravity", Phys. Rev. D59 (1999) 046006 hep-th/9808061.

[30] B.E.W. Nilsson and A.K. Tollstén, "Superspace formulation of the ten-dimensional coupled Einstein-YangMills system", Phys. Lett. B171 (1986) 212.

[31] I. Pesando and A.K. Tollstén, "Heterotic instantons and solitons in anomaly-free supergravity", Phys. Lett. B274 (1992) 374.

[32] A. Candiello and K. Lechner, "Duality in supergravity theories", Nucl. Phys. B412 (1994) 479.

[33] P. Howe, "Weyl superspace", Phys. Lett. B415 (1997) 149 hep-th/9707184].

[34] S.J. Gates, K.S. Stelle and P.C. West, "Algebraic origins of superspace constraints in supergravity", Nucl. 
Cederwall, Gran, Nielsen, Nilsson: "Manifestly Supersymmetric M-Theory" ......... 14

Phys. B169 (1980) 347; S.J. Gates and W. Siegel, "Understanding constraints in superspace formulation of supergravity", Nucl. Phys. B163 (1980) 519 .

[35] P.S. Howe, E. Sezgin and P.C. West, "Aspects of superembeddings", hep-th/9705093.

[36] S.J. Gates and H. Nishino, "Toward an off-shell 11-D supergravity limit of $M$ theory", Phys. Lett. B388 (1996) 504 hep-th/9602011.

[37] B.E.W. Nilsson, "Simple 10-dimensional supergravity in superspace", Nucl. Phys. B188 (1981) 176.

[38] M. Cederwall, U. Gran, M. Nielsen and B.E.W. Nilsson, in preparation.

[39] U. Gran, in preparation.

[40] B.E.W. Nilsson, "Off-shell $d=10, N=1$ Poincaré supergravity and the embeddibility of higher derivative field theories in superspace", Phys. Lett. B175 (1986) 319.

[41] A.M. Cohen, M. van Leeuwen and B. Lisser, LiE v. 2.2 (1998), http://wallis.univ-poitiers.fr/ ${ }^{\sim}$ maavl/LiE/ 